Freiburg-THEP 99/10

hep-ph/9909506

September 1999

\title{
Non-planar massless two-loop Feynman diagrams with four on-shell legs
}

\author{
J.B. Tausk" \\ Fakultät für Physik, Albert-Ludwigs-Universität Freiburg, \\ Hermann-Herder-Straße 3, D-79104 Freiburg, Germany
}

\begin{abstract}
The non-planar Feynman diagram with seven massless, scalar propagators and four on-shell legs (the crossed double box) is calculated analytically in dimensional regularization. The non-planar diagram with six propagators is also discussed.
\end{abstract}

\section{Introduction}

Knowledge of two-loop massless Feynman diagrams with four massless external legs is one of the key ingredients required for a next-to-next-to-leading order calculation of 2 jet production rates in hadron-hadron collisions [1], as well as for other processes, including Bhabha scattering in the high energy limit. Among those diagrams, the most complicated are the planar and non-planar double box diagrams, both of which contain seven propagators. Recently, significant progress in this field has been made by Smirnov, who has derived an analytical formula in terms of polylogarithms for the planar double box [2] (see also ref. [3]). More simple planar diagrams, with less than seven propagators, are discussed in refs. 四, 司. Results for non-planar diagrams have until now been missing.


The ultraviolet divergences of the planar and non-planar double box diagrams in spacetime dimensions $d=7, d=9$ and $d=11$ were calculated in ref. [6]. In $d=4$, the diagrams are ultraviolet finite, but they have a complicated structure of collinear and infrared divergences. In dimensional regularization, with $d=4-2 \epsilon$, the divergences appear as poles $1 / \epsilon^{j}$, with $j=1,2,3,4$. As has been demonstrated by Smirnov in the planar case, the method of Mellin-Barnes contour integrals is a very natural and convenient technique to

\footnotetext{
${ }^{1}$ tausk@physik.uni-freiburg.de
} 
isolate these singular contributions. The main purpose of this paper is to calculate the non-planar seven propagator diagram,

$$
B_{7}^{N P}=\iint \mathrm{d}^{d} k \mathrm{~d}^{d} l \frac{1}{\left(k+l+p_{1}+p_{2}\right)^{2}\left(k+l+p_{2}\right)^{2}(k+l)^{2}\left(k-p_{3}\right)^{2} k^{2}\left(l-p_{4}\right)^{2} l^{2}},
$$

using a similar Mellin-Barnes approach. The external momenta $p_{1} \ldots p_{4}$ are lightlike $\left(p_{i}^{2}=0\right)$ and we use Mandelstam variables defined by $s=\left(p_{1}+p_{2}\right)^{2}, t=\left(p_{1}+p_{3}\right)^{2}$ and $u=\left(p_{1}+p_{4}\right)^{2}$.

Starting from a Mellin-Barnes representation, which we derive in section 2, we obtain analytical formulas in terms of logarithms and polylogarithms for the seven propagator diagram $B_{7}^{N P}$, which we present in section 5 , by a series of steps that are explained in sections 3 and 4 . Then, in section 6 , we briefly discuss the non-planar diagram with six propagators. Some final comments are made in section 7 .

\section{A Mellin-Barnes representation}

Our main tool will be the Mellin-Barnes representation of a power of a sum as a contour integral,

$$
\left(A_{1}+A_{2}\right)^{-\nu}=\frac{1}{2 \pi i} \int_{-i \infty}^{i \infty} \mathrm{d} \sigma A_{1}^{\sigma} A_{2}^{-\nu-\sigma} \frac{\Gamma(-\sigma) \Gamma(\nu+\sigma)}{\Gamma(\nu)},
$$

where the integration contour separates the poles of $\Gamma(-\sigma)$ from those of $\Gamma(\nu+\sigma)$, and $A_{1,2}$ are complex numbers such that $\left|\arg \left(A_{1}\right)-\arg \left(A_{2}\right)\right|<\pi$. The Mellin-Barnes representation of a sum of several terms, $\left(A_{1}+\ldots+A_{n}\right)^{-\nu}$, is obtained easily by iteration of the basic formula (2). Another formula that we will need is Barnes's first lemma (see, e.g. [7]):

$$
\begin{array}{r}
\frac{1}{2 \pi i} \int_{-i \infty}^{i \infty} \mathrm{d} \sigma \Gamma\left(\sigma_{1}+\sigma\right) \Gamma\left(\sigma_{2}+\sigma\right) \Gamma\left(\sigma_{3}-\sigma\right) \Gamma\left(\sigma_{4}-\sigma\right) \\
=\frac{\Gamma\left(\sigma_{1}+\sigma_{3}\right) \Gamma\left(\sigma_{1}+\sigma_{4}\right) \Gamma\left(\sigma_{2}+\sigma_{3}\right) \Gamma\left(\sigma_{2}+\sigma_{4}\right)}{\Gamma\left(\sigma_{1}+\sigma_{2}+\sigma_{3}+\sigma_{4}\right)} .
\end{array}
$$

Again, the contour should separate the increasing series of poles (of $\Gamma\left(\sigma_{3,4}-\sigma\right)$ ) from the decreasing ones (of $\left.\Gamma\left(\sigma_{1,2}+\sigma\right)\right)$.

Introducing Feynman parameters in the standard way, the non-planar double box diagram can be written as [6]

$$
\begin{aligned}
B_{7}^{N P}= & (-1)^{7}\left(i \pi^{d / 2}\right)^{2} \Gamma(7-d) \int_{0}^{\infty} \mathrm{d} x_{1} \ldots \mathrm{d} x_{7} \delta\left(1-x_{1} \ldots-x_{7}\right) D^{7-\frac{3 d}{2}} C^{d-7} \\
D= & \left(x_{1}+x_{2}+x_{3}\right)\left(x_{4}+x_{5}+x_{6}+x_{7}\right)+\left(x_{4}+x_{5}\right)\left(x_{6}+x_{7}\right) \\
C= & -t x_{2} x_{5} x_{6}-u x_{2} x_{4} x_{7}-s x_{1} x_{5} x_{7}-s x_{3} x_{4} x_{6} \\
& -s x_{1} x_{3}\left(x_{4}+x_{5}+x_{6}+x_{7}\right) .
\end{aligned}
$$

According to the causal $i \epsilon$-prescription, a small imaginary part should be added to $C$. We do this by giving $s, t$ and $u$ positive imaginary parts. At this stage, we consider $s$, $t$ and $u$ to be three independent variables. At the end of the calculation, after we have taken the limit where $s, t$ and $u$ become real, one variable can be eliminated by using the relationship $s+t+u=0$. 
The next step is to represent the factor $C^{d-7}$ as a product of five more simple factors, at the expense of introducing four Mellin-Barnes parameters:

$$
\begin{aligned}
\Gamma(7-d) C^{d-7}= & \frac{1}{(2 \pi i)^{4}} \int_{-i \infty}^{i \infty} \mathrm{d} \xi_{1} \int_{-i \infty}^{i \infty} \mathrm{d} \xi_{2} \int_{-i \infty}^{i \infty} \mathrm{d} \alpha \int_{-i \infty}^{i \infty} \mathrm{d} \beta \\
& \times \Gamma\left(-\xi_{1}\right) \Gamma\left(-\xi_{2}\right) \Gamma(-\alpha) \Gamma(-\beta) \Gamma\left(7-d+\xi_{1}+\xi_{2}+\alpha+\beta\right) \\
& \times\left[-t x_{2} x_{5} x_{6}\right]^{\xi_{1}}\left[-u x_{2} x_{4} x_{7}\right]^{\xi_{2}}\left[-s x_{1} x_{5} x_{7}\right]^{\alpha}\left[-s x_{3} x_{4} x_{6}\right]^{\beta} \\
& \times\left[-s x_{1} x_{3}\left(x_{4}+x_{5}+x_{6}+x_{7}\right)\right]^{d-7-\xi_{1}-\xi_{2}-\alpha-\beta} .
\end{aligned}
$$

Now the Feynman parameter integrals can be done in terms of $\Gamma$-functions, and we obtain the following Mellin-Barnes representation for $B_{7}^{N P}$ :

$$
\begin{gathered}
B_{7}^{N P}=\frac{\pi^{d} \Gamma^{2}(-\epsilon)}{\Gamma(-2 \epsilon) \Gamma(-1-3 \epsilon)} K \\
K=\frac{1}{(2 \pi i)^{4}} \int_{-i \infty}^{i \infty} \mathrm{d} \xi_{1} \int_{-i \infty}^{i \infty} \mathrm{d} \xi_{2} \int_{-i \infty}^{i \infty} \mathrm{d} \alpha \int_{-i \infty}^{i \infty} \mathrm{d} \beta \\
\times(-s)^{-3-2 \epsilon-\xi_{1}-\xi_{2}}(-t)^{\xi_{1}}(-u)^{\xi_{2}} \frac{\Gamma_{1} \Gamma_{2} \Gamma_{3} \Gamma_{4} \Gamma_{5} \Gamma_{6} \Gamma_{7} \Gamma_{8} \Gamma_{9} \Gamma_{10} \Gamma_{11} \Gamma_{12} \Gamma_{13}}{\Gamma_{14}^{2}}
\end{gathered}
$$

where

$$
\begin{array}{ll}
\Gamma_{1}=\Gamma\left(3+2 \epsilon+\alpha+\beta+\xi_{1}+\xi_{2}\right) & \Gamma_{8}=\Gamma\left(-2-2 \epsilon-\alpha-\xi_{1}-\xi_{2}\right) \\
\Gamma_{2}=\Gamma\left(2+\epsilon+\alpha+\beta+\xi_{1}+\xi_{2}\right) & \Gamma_{9}=\Gamma\left(-2-2 \epsilon-\beta-\xi_{1}-\xi_{2}\right) \\
\Gamma_{3}=\Gamma\left(1+\xi_{1}+\xi_{2}\right) & \Gamma_{10}=\Gamma(-\alpha) \\
\Gamma_{4}=\Gamma\left(1+\alpha+\xi_{1}\right) & \Gamma_{11}=\Gamma(-\beta) \\
\Gamma_{5}=\Gamma\left(1+\alpha+\xi_{2}\right) & \Gamma_{12}=\Gamma\left(-\xi_{1}\right) \\
\Gamma_{6}=\Gamma\left(1+\beta+\xi_{1}\right) & \Gamma_{13}=\Gamma\left(-\xi_{2}\right) \\
\Gamma_{7}=\Gamma\left(1+\beta+\xi_{2}\right) & \Gamma_{14}=\Gamma\left(2+\alpha+\beta+\xi_{1}+\xi_{2}\right) .
\end{array}
$$

In order to keep things as simple as possible, we choose the contour for each of the variables $\xi_{1}, \xi_{2}, \alpha$ and $\beta$ to be a straight line parallel to the imaginary axis, with a real part that does not depend on any of the other variables. Eq. (9) is certainly valid if the real parts of the contours are chosen in such a way that the real parts of the arguments of $\Gamma_{1} \ldots \Gamma_{13}$ are all positive. It is possible to satisfy these conditions simultaneously when $-3 / 2<\Re(\epsilon)<-1 / 3$. For example, if $\epsilon \approx-1 / 2$, choosing $\Re\left(\xi_{1}\right)=\Re\left(\xi_{2}\right)=-5 / 12$, $\Re(\alpha)=\Re(\beta)=-1 / 4$, satifies all the conditions. Obviously, there are infinitely many other, equally suitable possibilities, but for the sake of definiteness, we will stick to these contours.

\section{Analytic continuation in $\epsilon$}

Having derived the Mellin-Barnes representation (9) near $\epsilon=-1 / 2$, we must now perform an analytic continuation in $\epsilon$ to the region around $\epsilon=0$. To do so, we follow the poles of the $\Gamma$-functions as we increase $\epsilon$. Whenever a pole crosses one of the integration contours, which we keep fixed, we add its residue in the corresponding variable to the right hand side of eq. (9). This residue term still contains poles in its remaining variables which can cross other integration contours as $\epsilon$ is increased further. They are treated in the same 
way. We end up with a large collection of residue terms, plus the original fourfold integral. Some of the residue terms contain factors that diverge at $\epsilon=0$. However, these singular factors do not depend on $\xi_{1}, \xi_{2}, \alpha$ or $\beta$. The remaining integrals can simply be expanded in $\epsilon$ under the integral sign because our contours do not go through any poles at $\epsilon=0$. (There are never problems with convergence at the limits $\xi_{1}, \xi_{2}, \alpha, \beta \rightarrow \pm i \infty$ ).

Below, we will give some examples of the residue terms that appear. But first, let us see what happens when we substitute $\epsilon=0$ in the integral (91). The variables $\alpha$ and $\beta$ are coupled via $\Gamma_{1}, \Gamma_{2}$ and $\Gamma_{14}$, but these $\Gamma$-functions collapse into

$$
\Gamma_{1} \Gamma_{2} / \Gamma_{14}^{2}=2+\alpha+\beta+\xi_{1}+\xi_{2}=\left(1+\alpha+\xi_{1}\right)+\left(1+\beta+\xi_{2}\right) .
$$

Consider the first term. The factor $\left(1+\alpha+\xi_{1}\right)$ can be absorbed into $\Gamma_{4}$, and then both the $\alpha$ and the $\beta$ integrals can be performed using Barnes's first lemma. Moreover, the $\beta$ integral gives zero:

$$
\begin{aligned}
\frac{1}{2 \pi i} \int_{-i \infty}^{i \infty} \mathrm{d} \beta \Gamma\left(1+\beta+\xi_{1}\right) \Gamma\left(1+\beta+\xi_{2}\right) \Gamma\left(-2-\beta-\xi_{1}-\xi_{2}\right) \Gamma(-\beta) \\
=\frac{\Gamma\left(-1-\xi_{1}\right) \Gamma\left(-1-\xi_{2}\right) \Gamma\left(1+\xi_{1}\right) \Gamma\left(1+\xi_{2}\right)}{\Gamma(0)}=0 .
\end{aligned}
$$

(Here, we are taking a contour that separates the increasing and decreasing series of poles as required by Barnes's lemma, not a straight line). Analogously, the second term on the right hand side of eq. (11) vanishes when integrated over $\alpha$.

This cancellation is very nice because it means that if we only need the non-planar box up to the constant term in $\epsilon$, once we have done the $\alpha$ and $\beta$ integrals, we do not need to calculate any double integrals of the form

$$
\int_{-i \infty}^{i \infty} \mathrm{d} \xi_{1} \int_{-i \infty}^{i \infty} \mathrm{d} \xi_{2}(-s)^{-3-\xi_{1}-\xi_{2}}(-t)^{\xi_{1}}(-u)^{\xi_{2}} \ldots
$$

Only terms in which a residue has been taken in either $\xi_{1}$ or $\xi_{2}$, or in both, are ever needed. Such terms, whose dependence on at least one of the variables $s, t$ and $u$ is trivial, are much more simple than the double integrals (13).

We now return to the task of collecting the various residue contributions. The first poles to cross contours are at $\alpha=-2-2 \epsilon-\xi_{1}-\xi_{2}$ and $\beta=-2-2 \epsilon-\xi_{1}-\xi_{2}$, coming from $\Gamma_{8}$ and $\Gamma_{9}$, respectively. (The poles of $\Gamma_{1}$ and $\Gamma_{2}$ are harmless because they depend on $\epsilon$ with the opposite sign, and therefore move away from the contours when $\Re(\epsilon)$ increases). To account for the residues of these two poles, we replace $K \rightarrow K+K_{8}+K_{9}+K_{89}$, where

$$
\begin{aligned}
K_{8}= & \frac{1}{(2 \pi i)^{3}} \int_{-i \infty}^{i \infty} \mathrm{d} \xi_{1} \int_{-i \infty}^{i \infty} \mathrm{d} \xi_{2} \int_{-i \infty}^{i \infty} \mathrm{d} \beta(-s)^{-3-2 \epsilon-\xi_{1}-\xi_{2}}(-t)^{\xi_{1}}(-u)^{\xi_{2}} \\
& \times\left.\frac{\Gamma_{1} \Gamma_{2} \Gamma_{3} \Gamma_{4} \Gamma_{5} \Gamma_{6} \Gamma_{7} \Gamma_{9} \Gamma_{10} \Gamma_{11} \Gamma_{12} \Gamma_{13}}{\Gamma_{14}^{2}}\right|_{\alpha=-2-2 \epsilon-\xi_{1}-\xi_{2}},
\end{aligned}
$$

$K_{9}$ is a similar term, where the residue in $\beta$ is taken, and

$$
\begin{aligned}
K_{89}= & \frac{1}{(2 \pi i)^{2}} \int_{-i \infty}^{i \infty} \mathrm{d} \xi_{1} \int_{-i \infty}^{i \infty} \mathrm{d} \xi_{2}(-s)^{-3-2 \epsilon-\xi_{1}-\xi_{2}}(-t)^{\xi_{1}}(-u)^{\xi_{2}} \\
& \times\left.\frac{\Gamma_{1} \Gamma_{2} \Gamma_{3} \Gamma_{4} \Gamma_{5} \Gamma_{6} \Gamma_{7} \Gamma_{10} \Gamma_{11} \Gamma_{12} \Gamma_{13}}{\Gamma_{14}^{2}}\right|_{\alpha=\beta=-2-2 \epsilon-\xi_{1}-\xi_{2}}
\end{aligned}
$$


Let us concentrate on $K_{8}$. Note that in eliminating $\alpha$, we have changed the $\epsilon$-dependence of the remaining $\Gamma$-functions. As a consequence, the first poles of $\Gamma_{2}=\Gamma(\beta-\epsilon), \Gamma_{4}=$ $\Gamma\left(-1-2 \epsilon-\xi_{2}\right)$ and $\Gamma_{5}=\Gamma\left(-1-2 \epsilon-\xi_{1}\right)$ cross the $\beta, \xi_{2}$ and $\xi_{1}$ contours, respectively. These crossings are taken into account by replacing $K_{8} \rightarrow K_{8}+K_{82}+K_{84}+K_{85}+$ $K_{842}+K_{852}+K_{845}+K_{8452}$. In a number of these terms, there are still more poles that cross contours before $\epsilon$ finally reaches 0 . They are dealt with by making the following further replacements: $K_{82} \rightarrow K_{82}+K_{829^{\prime}}, K_{84} \rightarrow K_{84}+K_{843}, K_{842} \rightarrow K_{842}+K_{8423}$, $K_{85} \rightarrow K_{85}+K_{853}, K_{852} \rightarrow K_{852}+K_{8523}$, and $K_{845} \rightarrow K_{845}-K_{8459}$. In each term, the subscripts indicate the $\Gamma$-functions of which the residue of the first pole has been taken. An exception to this is $K_{829^{\prime}}$, where the prime means that we have taken the residue of the second pole of $\Gamma_{9}$ (i.e., where the argument equals -1 ) instead of the first pole. The last term, $K_{8459}$, gets a relative minus sign because the pole in $\Gamma_{9}$ crosses the $\beta$ contour backwards.2]

Due to the symmetry of the problem in $\alpha$ and $\beta$, there is a one to one correspondence between the descendants of $K_{9}$ and those of $K_{8}$, and their contributions to the final result are the same. The analysis of $K_{89}$ runs along similar lines. Here, some poles are second order, with residues involving derivatives of $\Gamma$-functions.

\section{Evaluating the $K$ 's}

In all, we find 39 contributions. (This number depends on the contours one uses. Had we chosen different ones, there would have been other poles crossing contours and we would have obtained a different, but equivalent, collection of residue terms). The simplest contributions are those where a residue in four variables has been taken, e.g.

$$
\begin{gathered}
K_{8452}=(-s)^{-3-2 \epsilon-\xi_{1}-\xi_{2}}(-t)^{\xi_{1}}(-u)^{\xi_{2}} \frac{\Gamma_{1} \Gamma_{3} \Gamma_{6} \Gamma_{7} \Gamma_{9} \Gamma_{10} \Gamma_{11} \Gamma_{12} \Gamma_{13}}{\Gamma_{14}^{2}} \mid \begin{array}{c}
\xi_{1}=\xi_{2}=-1-2 \epsilon \\
\alpha=2 \epsilon, \beta=\epsilon
\end{array} \\
=(-s)^{-1+2 \epsilon}(-t)^{-1-2 \epsilon}(-u)^{-1-2 \epsilon} \Gamma(1+\epsilon) \Gamma(-1-4 \epsilon) \Gamma(\epsilon) \Gamma(-2 \epsilon) \Gamma(-\epsilon) \Gamma^{2}(1+2 \epsilon) .
\end{gathered}
$$

Note that in this particular case, the $\epsilon$ expansion starts off with a $1 / \epsilon^{4}$ pole.

In several terms, there is one integral left. For instance, $K_{843}$, which we get by taking residues at $\alpha=-2-2 \epsilon-\xi_{1}-\xi_{2}, \xi_{2}=-1-2 \epsilon$ and $\xi_{1}=2 \epsilon$, is given by

$$
\begin{aligned}
& K_{843}=(-s)^{-2-2 \epsilon}(-t)^{2 \epsilon}(-u)^{-1-2 \epsilon} \Gamma(-1-4 \epsilon) \Gamma^{2}(1+2 \epsilon) \Gamma(-2 \epsilon) \\
& \times \frac{1}{2 \pi i} \int_{-i \infty}^{i \infty} \mathrm{d} \beta \frac{\Gamma(1+\beta) \Gamma(\beta-\epsilon) \Gamma(1+2 \epsilon+\beta) \Gamma(-1-2 \epsilon-\beta) \Gamma(-\beta)}{\Gamma(\beta-2 \epsilon)} .
\end{aligned}
$$

To calculate the $\beta$ integral, we expand the integrand up to the second order in $\epsilon$. Then, closing the contour to the left or right and summing the residues of the poles inside it, we obtain harmonic series which can be expressed in terms of $\zeta(2), \zeta(3)$ and $\zeta(4)$.

An example in which the final integral still involves powers of Mandelstam variables is

$$
K_{842}=\frac{-1}{2 \pi i u} \int_{-i \infty}^{i \infty} \mathrm{d} \xi_{1}(-s)^{-2-\xi_{1}}(-t)^{\xi_{1}} \frac{\Gamma^{3}\left(1+\xi_{1}\right) \Gamma^{3}\left(-\xi_{1}\right)}{\xi_{1}\left(1+\xi_{1}\right)^{2}},
$$

where we have already set $\epsilon=0$. This integral can also be calculated by closing the contour, and the result can be expressed in terms of polylogarithms $\mathrm{Li}_{n}$ [8] and Nielsen's

\footnotetext{
${ }^{2}$ Eventually, $K_{8459}$ cancels against a descendant of $K_{89}$.
} 
generalized polylogarithms $\mathrm{S}_{n, p}$ [9, 10], which are defined by

$$
\mathrm{S}_{n, p}(z)=\frac{(-1)^{n-1+p}}{(n-1) ! p !} \int_{0}^{1} \mathrm{~d} t \frac{\log ^{n-1}(t) \log ^{p}(1-z t)}{t}
$$

The cases $\mathrm{S}_{1,2}, \mathrm{~S}_{1,3}$ and $\mathrm{S}_{2,2}$ which occur in this paper can all be written as combinations of $\mathrm{Li}_{2}, \mathrm{Li}_{3}$ and $\mathrm{Li}_{4}$.

In the term $K_{84}$, we perform the first integration by Barnes's lemma (in a slightly modified form, see [2]):

$$
\begin{aligned}
K_{84}= & \frac{1}{(2 \pi i)^{2} u} \int_{-i \infty}^{i \infty} \mathrm{d} \xi_{1}(-s)^{-2-\xi_{1}}(-t)^{\xi_{1}} \frac{\Gamma^{2}\left(1+\xi_{1}\right) \Gamma^{2}\left(-\xi_{1}\right)}{\xi_{1}\left(1+\xi_{1}\right)} \\
& \times \int_{-i \infty}^{i \infty} \mathrm{d} \beta \Gamma(1+\beta) \Gamma\left(1+\beta+\xi_{1}\right) \Gamma\left(-1-\beta-\xi_{1}\right) \Gamma(-\beta) \\
= & \frac{1}{2 \pi i u} \int_{-i \infty}^{i \infty} \mathrm{d} \xi_{1}(-s)^{-2-\xi_{1}}(-t)^{\xi_{1}} \frac{\Gamma^{3}\left(1+\xi_{1}\right) \Gamma^{3}\left(-\xi_{1}\right)}{\xi_{1}\left(1+\xi_{1}\right)}\left(\psi\left(1+\xi_{1}\right)-\psi(1)\right) .
\end{aligned}
$$

The $\xi_{1}$ integral can be then be done by closing the contour, as in the previous example. Three other $K$ 's are related to $K_{84}$ by $\alpha \leftrightarrow \beta$ and/or $\xi_{1} \leftrightarrow \xi_{2}$.

Finally, there are four terms in which both the $\xi_{1}$ and the $\xi_{2}$ integrals still survive, namely $K, K_{8}, K_{9}$ and $K_{89}$. However, by the mechanism explained above (see eq. (12)), these terms cancel against each other at $\epsilon=0$ after integration over $\alpha$ and $\beta$.

\section{Result for the seven propagator diagram}

Adding together all the contributions discussed in the previous section, we obtain an expression for $B_{7}^{N P}$, up to the constant term in $\epsilon$, in terms of (poly)logarithms of (ratios of) $s, t$ and $u$, which are, at this point, still independent variables. In particular, the result is also valid when $s, t$ and $u$ are all negative. This region is, of course, unphysical, but it is nevertheless interesting to consider because it provides us with an opportunity to check our result against a calculation by Binoth and Heinrich [11]. They have obtained results for the negative $s, t, u$ region by extracting the divergences from the Feynman parametric integral (4) and then performing a multi-dimensional numerical integration.

At the symmetric point, $s=t=u=-1$, we find

$$
\begin{aligned}
& B_{7}^{N P}=\pi^{d} \Gamma(3+2 \epsilon)\left\{\frac{7}{4 \epsilon^{4}}-\frac{3}{\epsilon^{3}}+\frac{1}{\epsilon^{2}}\left(\frac{-7}{2}-\frac{47}{24} \pi^{2}\right)+\frac{1}{\epsilon}\left(\frac{105}{2}+\frac{37}{4} \pi^{2}-\frac{89}{4} \zeta(3)\right.\right. \\
& \left.-\frac{1}{2} \pi^{2} \log (2)\right)-\frac{589}{2}-\frac{112}{3} \pi^{2}-\frac{443}{288} \pi^{4}+\frac{555}{4} \zeta(3) \\
& \left.+\frac{75}{2} \pi^{2} \log (2)-\frac{11}{3} \pi^{2} \log ^{2}(2)+\frac{11}{12} \log ^{4}(2)+22 \operatorname{Li}_{4}(1 / 2)\right\} \\
& =\pi^{d} \Gamma(3+2 \epsilon)\left\{\frac{1.75}{\epsilon^{4}}-\frac{3}{\epsilon^{3}}-\frac{22.828}{\epsilon^{2}}+\frac{113.63}{\epsilon}-395.26\right\} \text {. }
\end{aligned}
$$

The coefficients in (21) agree with the results obtained by the numerical method [11]. We also find good agreement at the asymmetric point $(s, t, u)=(-1,-2,-3)$. In both cases

\footnotetext{
${ }^{3}$ We are leaving out $K_{82}$ and $K_{92}$, which contain a factor of $\Gamma(-\epsilon)$ in their denominator and are therefore already of order $\epsilon$.
} 
the precision of the numerical method ranges from about 1 per mille for the $1 / \epsilon^{4}$-term to 1 per cent for the constant term.

Since the complete formula for general $s, t$ and $u$ is very long and not relevant for physical applications anyway, we do not present it here. Instead, we specialize to the physically relevant case where $s+t+u=0$. We also use transformation formulas for the arguments of the polylogarithms in order to avoid ending up on a cut when we make $s$, $t$, and $u$ real. Depending on which legs correspond to incoming and outgoing particles, there are three different kinematical regions to consider: (i), where $u, t<0<s$; (ii), where $u, s<0<t$; and (iii), where $s, t<0<u$. In all regions, we have

$$
B_{7}^{N P}=\left(i \pi^{d / 2}\right)^{2} \Gamma^{2}(1+\epsilon)\left\{\frac{F_{t}}{s^{2} t}+\frac{F_{u}}{s^{2} u}\right\}
$$

We will use the abbreviations $T=\log (-t / s), U=\log (-u / s), V=\log (-u / t)$.

In region (i), with $u, t<0$, and $s=-t-u, F_{t}$ is given by

$$
\begin{aligned}
F_{t}= & s^{-2 \epsilon}\left\{\frac{-2}{\epsilon^{4}}+\frac{1}{2 \epsilon^{3}}(5 T+7 U)+\frac{1}{\epsilon^{2}}\left(6 U-T^{2}-4 T U-U^{2}-\frac{5}{12} \pi^{2}\right)\right. \\
+ & \frac{1}{\epsilon}\left(-24 U-12 T U-\frac{1}{3} T^{3}+3 T U^{2}-U^{3}+\frac{1}{6} \pi^{2}(5 T-29 U)\right. \\
& \left.\quad-2 T \mathrm{Li}_{2}(-t / s)+2 \mathrm{Li}_{3}(-t / s)-2 \mathrm{~S}_{1,2}(-t / s)+\frac{19}{2} \zeta(3)\right) \\
+ & 96 U+48 T U-12 T^{2} U+12 T U^{2}-4 U^{3}+\frac{2}{3} T^{4}+\frac{8}{3} T^{3} U-T^{2} U^{2} \\
- & \frac{4}{3} T U^{3}+\frac{4}{3} U^{4}+\pi^{2}\left(-14 U-\frac{5}{6} T^{2}+\frac{38}{3} T U+\frac{25}{6} U^{2}\right)+\frac{37}{40} \pi^{4} \\
- & (13 T+33 U) \zeta(3)+\left(-48 T+17 T^{2}+12 T U+10 \pi^{2}\right) \mathrm{Li}_{2}(-t / s) \\
+ & (48-60 T-12 U) \mathrm{Li}_{3}(-t / s)+(-24+28 T-6 U) \mathrm{S}_{1,2}(-t / s) \\
+ & 86 \mathrm{Li}_{4}(-t / s)-26 \mathrm{~S}_{1,3}(-t / s)-36 \mathrm{~S}_{2,2}(-t / s) \\
+ & i \pi\left[\frac{2}{\epsilon^{3}}+\frac{1}{\epsilon^{2}}(6-T+U)-\frac{1}{\epsilon}\left(24+12 T+2 T^{2}+2 T U+2 U^{2}+\frac{31}{6} \pi^{2}\right)\right. \\
& +96+48 T+12 T^{2}-24 T U+\frac{10}{3} T^{3}+2 U^{3} \\
& +\pi^{2}\left(-2+\frac{28}{3} T-\frac{1}{3} U\right)+(-24+14 T+18 U) \mathrm{Li}_{2}(-t / s) \\
& \left.\left.-32 \mathrm{Li}_{3}(-t / s)+44 \mathrm{~S}_{1,2}(-t / s)-89 \zeta(3)\right]\right\}
\end{aligned}
$$

and $F_{u}$ can be obtained by interchanging $(t \leftrightarrow u)$ in the expression for $F_{t}$.

In region (ii), with $u, s<0$, and $t=-s-u, F_{t}$ and $F_{u}$ are given by

$$
\begin{gathered}
F_{t}=t^{-2 \epsilon}\left\{\frac{-2}{\epsilon^{4}}+\frac{1}{\epsilon^{3}}\left(2 T+\frac{7}{2} V\right)+\frac{1}{\epsilon^{2}}\left(6 T+6 V+2 T^{2}+T V-V^{2}+\frac{31}{12} \pi^{2}\right)\right. \\
+\frac{1}{\epsilon}\left(-24 T-24 V-\frac{2}{3} T^{3}-2 T^{2} V-2 T V^{2}-V^{3}-\frac{1}{6} \pi^{2}(23 T+41 V)\right. \\
\left.+2 \mathrm{~S}_{1,2}(-s / t)+\frac{15}{2} \zeta(3)\right)
\end{gathered}
$$




$$
\begin{aligned}
& +96 T+96 V-4 T^{3}-12 T^{2} V-4 V^{3}-\frac{11}{6} T^{4}-\frac{13}{3} T^{3} V+T^{2} V^{2}+2 T V^{3}+\frac{4}{3} V^{4} \\
& +\pi^{2}\left(-14 T-14 V-\frac{22}{3} T^{2}-\frac{23}{3} T V+\frac{37}{6} V^{2}\right)-\frac{311}{120} \pi^{4} \\
& -(24+45 T+39 V) \zeta(3)+\left(24 T+6 T^{2}-18 T V+13 \pi^{2}\right) \mathrm{Li}_{2}(-s / t) \\
& +(24+12 T-18 V) \mathrm{Li}_{3}(-s / t)+(24-44 T+6 V) \mathrm{S}_{1,2}(-s / t) \\
& +12 \mathrm{Li}_{4}(-s / t)+26 \mathrm{~S}_{1,3}(-s / t)-62 \mathrm{~S}_{2,2}(-s / t) \\
& +i \pi\left[\frac{-5}{2 \epsilon^{3}}+\frac{1}{\epsilon^{2}}(T+4 V)\right. \\
& +\frac{1}{\epsilon}\left(12 T+12 V+4 T^{2}+2 T V-3 V^{2}+\frac{5}{2} \pi^{2}-2 \mathrm{Li}_{2}(-s / t)\right) \\
& -48 T-48 V+12 T^{2}+24 T V-12 V^{2}+\frac{11}{3} T^{3}+2 T^{2} V+\frac{4}{3} V^{3} \\
& +\pi^{2}(4-6 V)+(-48+14 T+12 V) \operatorname{Li}_{2}(-s / t) \\
& \left.\left.+32 \mathrm{Li}_{3}(-s / t)+28 \mathrm{~S}_{1,2}(-s / t)-15 \zeta(3)\right]\right\} \text {, } \\
& F_{u}=t^{-2 \epsilon}\left\{\frac{-2}{\epsilon^{4}}+\frac{1}{\epsilon^{3}}\left(2 T+\frac{5}{2} V\right)+\frac{1}{\epsilon^{2}}\left(6 T+2 T^{2}-T V-V^{2}+\frac{31}{12} \pi^{2}\right)\right. \\
& +\frac{1}{\epsilon}\left(-24 T-12 T V-\frac{2}{3} T^{3}-4 T^{2} V-2 T V^{2}-\frac{1}{3} V^{3}-\frac{1}{6} \pi^{2}(23 T+31 V)\right. \\
& \left.+2 \mathrm{~S}_{1,2}(-s / t)+\frac{15}{2} \zeta(3)\right) \\
& +96 T+48 T V-4 T^{3}+12 T V^{2}-\frac{11}{6} T^{4}-\frac{5}{3} T^{3} V+4 T^{2} V^{2}+\frac{10}{3} T V^{3}+\frac{2}{3} V^{4} \\
& +\pi^{2}\left(-14 T-\frac{22}{3} T^{2}-\frac{7}{3} T V+\frac{31}{6} V^{2}\right)-\frac{311}{120} \pi^{4}-(24+45 T+21 V) \zeta(3) \\
& +\left(-24 T-6 T^{2}-18 T V+11 \pi^{2}\right) \mathrm{Li}_{2}(-s / t)-(24+12 T+18 V) \mathrm{Li}_{3}(-s / t) \\
& +(48-32 T+26 V) \mathrm{S}_{1,2}(-s / t)-12 \mathrm{Li}_{4}(-s / t)+86 \mathrm{~S}_{1,3}(-s / t)-50 \mathrm{~S}_{2,2}(-s / t) \\
& +i \pi\left[\frac{-7}{2 \epsilon^{3}}+\frac{1}{\epsilon^{2}}(-6-T+4 V)\right. \\
& +\frac{1}{\epsilon}\left(24+12 V+2 T^{2}+2 T V-V^{2}+\frac{11}{2} \pi^{2}-2 \mathrm{Li}_{2}(-s / t)\right) \\
& -96-48 V-12 V^{2}+\frac{7}{3} T^{3}-4 T^{2} V-2 T V^{2}-\frac{4}{3} V^{3} \\
& +\pi^{2}(6+6 T-8 V)+(-24+26 T-8 V) \operatorname{Li}_{2}(-s / t) \\
& \left.\left.+44 \mathrm{Li}_{3}(-s / t)-24 \mathrm{~S}_{1,2}(-s / t)+75 \zeta(3)\right]\right\} \text {. }
\end{aligned}
$$

The formulas for $F_{t}$ and $F_{u}$ in region (iii) can be obtained easily from the ones for region (ii) by using the fact the $B_{7}^{N P}$ is symmetric under $(t \leftrightarrow u)$. This follows from the symmetry between the legs of the non-planar double box carrying momenta $p_{3}$ and $p_{4}$.

\section{Six propagator diagram}


The non-planar diagram with six propagators,

$$
B_{6}^{N P}=\iint \mathrm{d}^{d} k \mathrm{~d}^{d} l \frac{1}{\left(k+l+p_{2}\right)^{2}(k+l)^{2}\left(k-p_{3}\right)^{2} k^{2}\left(l-p_{4}\right)^{2} l^{2}},
$$

is far less difficult to calculate than $B_{7}^{N P}$. Perhaps the easiest way is by considering a scale transformation,

$$
B_{6}^{N P}\left(\lambda p_{1}, \lambda p_{2}, \lambda p_{3}, \lambda p_{4}\right)=\lambda^{2(d-6)} B_{6}^{N P}\left(p_{1}, p_{2}, p_{3}, p_{4}\right) .
$$

Differentiating both sides of eq. (27) with respect to $\lambda$ at $\lambda=1$ gives an identity which can be used to reduce $B_{6}^{N P}$ to a sum of three planar diagrams:

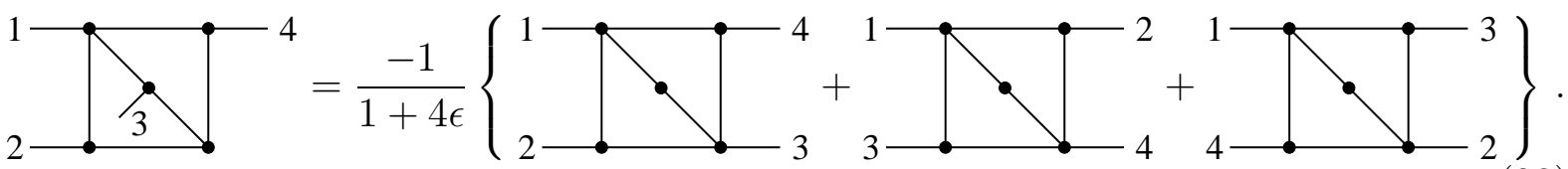

In the diagrams on the right hand side of eq. (28), the propagator on the diagonal is squared. Such boxes with a diagonal propagator are calculated in ref. [4]. They are, in fact, rather similar to one-loop massless box diagrams [12]. The pole at $\epsilon=-1 / 4$ is a reflection of the linear infrared divergencef in (26) coming from the region where the loop momenta $k$ and $l$ are both soft. Another curious feature of eq. (28) is that although the three terms on the right hand side contain third order poles at $\epsilon=0$, the leading singularity of the sum is only $1 / \epsilon^{2}$.

The six propagator diagram is completely symmetric under permutations of its external momenta. Therefore, it is sufficient to consider only region (i), where $u, t<0<s$ and $s=-t-u$. We obtain

$$
\begin{aligned}
B_{6}^{N P}= & \left(i \pi^{d / 2}\right)^{2} \frac{\Gamma(1+2 \epsilon) \Gamma^{3}(1-\epsilon)}{\Gamma(1-3 \epsilon)(1+4 \epsilon)} \frac{3}{\epsilon^{2}}\left\{\frac{s^{-2 \epsilon}}{s u}((T+i \pi)(1-2 \epsilon U)\right. \\
& +2 \epsilon^{2}\left(2 \mathrm{Li}_{3}(-t / s)-4 \mathrm{~S}_{1,2}(-t / s)+2 \zeta(3)-\frac{1}{3} T^{3}\right. \\
& \left.\left.+T\left[-2 \mathrm{Li}_{2}(-t / s)+U^{2}-\pi^{2}\right]+i \pi\left[2 \mathrm{Li}_{2}(-t / s)+U^{2}-\frac{1}{3} \pi^{2}\right]\right)\right) \\
& +(u \leftrightarrow t)\} .
\end{aligned}
$$

The reduction formula (28) can be generalized to the case where the six propagators in eq. (26) are raised to arbitrary integer powers.

\section{Discussion}

The analytical property that distinguishes the non-planar diagrams from the planar ones considered in refs. [2, 4, 5] is the fact that they have cuts in three channels, $s, t$, and $u$, rather than just in two. As a consequence, they have imaginary parts in all three physical regions, (i), (ii) and (iii). This is why, when we derived the Mellin-Barnes representation (9) for the seven propagator diagram, we were led to considering it as a function of

\footnotetext{
${ }^{4}$ It would diverge as $1 / m_{\text {reg }}$ if we used a mass $m_{\text {reg }}$ to regularize it.
} 
three independent variables. A priori, one would think that going from a two-scale problem to a three-scale problem could make it vastly more difficult to solve, but it did not, because, thanks to the vanishing of (12), all genuinely three-scale contributions cancelled out.

Generalizing the Mellin-Barnes representation for the seven propagator diagram to arbitrary powers of propagators is completely straightforward, and merely amounts to shifting the arguments of the $\Gamma$-functions (10) by some constants. It is likely that cancellations similar to (12) will take place for other integer powers, so that the method described in this paper could be used for those cases as well.

I am very grateful to T. Binoth and G. Heinrich for their help with the numerical checks, and for many discussions. I would also like to thank J.J. van der Bij, A.I. Davy-

dychev, T. Gehrmann, K. Melnikov, E. Remiddi and V.A. Smirnov for helpful discussions and correspondence. This work was supported by the DFG-Forschergruppe "Quantenfeldtheorie, Computeralgebra und Monte-Carlo-Simulation".

\section{References}

[1] E.W.N. Glover, hep-ph/9805481.

[2] V.A. Smirnov, Phys. Lett. B460 (1999), 397.

[3] N.I. Ussyukina, preprint FERMILAB-PUB-93/241-T;

N.I. Ussyukina, hep-ph/9801261.

[4] V.A. Smirnov and O.L. Veretin, hep-ph/9907385.

[5] C. Anastasiou, E.W.N. Glover and C. Oleari, hep-ph/9907523.

[6] Z. Bern et al., Nucl. Phys. B530 (1998) 401.

[7] L.J. Slater, Generalized Hypergeometric Functions (Cambridge University Press, 1966).

[8] L. Lewin, Polylogarithms and associated functions (North Holland, 1981).

[9] N. Nielsen, Nova Acta Leopoldina 90 (1909) 123.

[10] K.S. Kölbig, J.A. Mignaco and E. Remiddi, BIT 10 (1970) 38.

[11] T. Binoth and G. Heinrich, unpublished.

[12] W.L. van Neerven, Nucl. Phys. B268 (1986) 453. 